\title{
Inhibition of iNOS protects endothelial-dependent vasodilation in aged rats
}

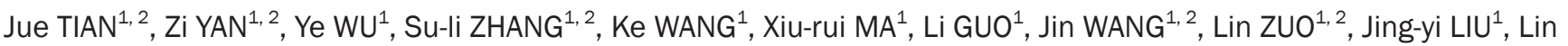 \\ QUAN $^{3}$, Hui-rong LIU $^{3,1, *}$ \\ ${ }^{1}$ Department of Physiology, Shanxi Medical University, Taiyuan 030001, China; ${ }^{2}$ State Key Laboratory of Cellular Physiology, Shanxi \\ Medical University, Taiyuan 030001, China; ${ }^{3}$ Department of Pathophysiology, School of Basic Medical Sciences, Capital Medical Uni- \\ versity, Beijing 100069, China
}

\begin{abstract}
Aim: To examine whether iNOS contributes to endothelial dysfunction in aged rats.
Methods: Male Sprague Dawley rats were divided into three groups: young rats, aged rats treated with vehicle and aged rats treated with N-[3-(Aminomethyl) benzyl] acetamidine (1400W, $1 \mathrm{mg} / \mathrm{kg}$, ip). Vasorelaxation was measured in isolated thoracic aorta. iNOS expression of thoracic aortic arteries was detected using immunohistochemistry and Western blot. Nitrotyrosine (a marker for peroxynitrite formation) content and expression in thoracic aortic tissue were determined using enzyme linked immunosorbent assay and immunohistochemistry.

Results: Maximal relaxation induced by acetylcholine $\left(10^{-9}\right.$ to $\left.10^{-5} \mathrm{~mol} / \mathrm{L}\right)$ in the aged rats treated with vehicle was significantly decreased $(70 \% \pm 15 \%, P<0.01)$, as compared with the young rats $(95 \% \pm 8 \%)$. However, the maximal relaxation induced by acidified NaNO2 (an endothelium-independent vasodilator) had no significant difference between the two groups. Moreover, iNOS and nitrotyrosine expression increased in the vessels of the aged rats. In the aged rats treated with 1400W (a highly selective iNOS inhibitor) nitrotyrosine expression was reduced and acetylcholine-induced vasorelaxation was markedly improved (maximal relaxation was increased to $87 \% \pm 8 \%, P<0.05$ ), but the acidified $\mathrm{NaNO}_{2}$-induced vasorelaxation had no significant change.

Conclusion: Our study demonstrated that inhibition of iNOS by $1400 \mathrm{~W}$ increased endothelium-dependent vasodilation in aged rats. The mechanism was related with attenuation of peroxynitrite formation.
\end{abstract}

Keywords: aging; nitric oxide synthase; endothelium; vasodilation; 1400W

Acta Pharmacologica Sinica (2010) 31: 1324-1328; doi: 10.1038/aps.2010.111; published online 13 Sep 2010

\section{Introduction}

With the further acceleration of aging, morbidity of various age-related cardiovascular diseases (such as hypertension, atherosclerosis, coronary heart disease, etc) is rising ${ }^{[1-3]}$, which not only reduced the quality of life of older persons but also increased the burden to the family and society. How to delay aging and promote the quality of older life has been a "hot" point in this field. Aging is a complex process caused by multiple factors ${ }^{[4]}$, in which the attenuation of endothelial vasodilatation has proven to be a striking feature ${ }^{[5]}$. It is well known that vascular endothelial dysfunction is a common trigger for many cardio-cerebral vascular diseases ${ }^{[6]}$, so protecting endothelial function is very important for prevention of agerelated cardiovascular diseases.

\footnotetext{
* To whom correspondence should be addressed.

E-mail liuhu2000@126.com

Received 2010-03-31 Accepted 2010-7-05
}

It has been reported that abnormal release of nitric oxide (NO) by endothelium is a major reason for the decline of endothelial-dependent vasodilatation ${ }^{[7-9]}$. Endogenous $\mathrm{NO}$ is generated by nitric oxide synthase (NOS) through catalyzing $L$-arginine. There are two kinds of NOS in blood vessels, endothelial NOS (eNOS) and inducible NOS (iNOS) ${ }^{[10-12]}$. Most previous studies suggested that decreased endothelialdependent vasodilatation was mainly associated with down regulation of eNOS and endogenous $\mathrm{NO}^{[13,14]}$. Recent research found that, iNOS expression increased in vascular tissue of aged rats ${ }^{[15,16]}$. However, it is unclear that whether elevated iNOS plays a role in the decline of endothelium-dependent vasodilatation.

Therefore, the purposes of the present study were to: (1) confirm that aging caused endothelial-dependent vasodilator dysfunction; (2) detect the level of iNOS in blood vessels; (3) clarify the role and possible mechanisms of iNOS in endothelial- dependent vasodilatation dysfunction. 


\section{Materials and methods}

Animals

The experimental procedures were conducted in adherence to the "Guiding Principles in the Use and Care of Animals" published by the National Institutes of Health (NIH Publication № 85-23, Revised 1996), the Guide for the Care and Use of Laboratory Animals protocol, published by the Ministry of the People's Republic of China (issued on 3rd June, 2004), and approved by the Institutional Animal Care and Use Committee of Shanxi Medical University. The experiments were performed in male Sprague Dawley rats [Grade II, certificate number of the breeder: SCXK(Jin) 2009-0001] in three groups ( $n=6-8$ rats per group): young (3-4 months old, weight 200$300 \mathrm{~g}$ ), aged (24-25 months old, weight 400-500 g) treated with vehicle (normal saline $0.5 \mathrm{~mL}$, ip) and aged treated with N-[3-(Aminomethyl) benzyl] acetamidine (1400W, Sigma, USA), a highly selective iNOS inhibitor $(1 \mathrm{mg} / \mathrm{kg} \text {, ip })^{[17,18]}$, respectively. Thirty minutes after $1400 \mathrm{~W}$ treatment ${ }^{[18,19]}$ young and aged rats were sacrificed to obtain vessel tissues $(n=7$ rats per group) for nitrotyrosine content and immunohistochemical studies of iNOS and nitrotyrosine.

\section{Determination of endothelial function}

Endothelial function was determined by comparing the vasorelaxation response to acetylcholine $(\mathrm{ACh})$, an endothelium-dependent vasodilator, with that of acidified $\mathrm{NaNO}_{2}$, an endothelium-independent vasodilator, as described previously ${ }^{[20]}$. Briefly, thoracic aortic rings were mounted onto hooks, suspended in organ chambers filled with Krebs buffer (mmol/L: $\mathrm{NaCl} 118.3, \mathrm{KCl} 4.7, \mathrm{CaCl}_{2} 2.5, \mathrm{MgSO}_{4} 1.2$, $\mathrm{KH}_{2} \mathrm{PO}_{4} 1.2, \mathrm{NaHCO}_{3} 25.0$, glucose 11.0, $\left.\mathrm{pH}=7.4\right)^{[21]}$ and aerated with $95 \% \mathrm{O}_{2}$ and $5 \% \mathrm{CO}_{2}$ at $37^{\circ} \mathrm{C}$, and connected to force transducers to record changes in tension via a Chart 5.3 data acquisition system. After equilibration for $60 \mathrm{~min}$ at a preload of $1 \mathrm{~g}$, the rings were precontracted with norepinephrine (NE, $1 \mu \mathrm{mol} / \mathrm{L})$. Once a stable contraction was achieved, the rings were exposed to cumulative concentrations of ACh $\left(10^{-9}\right.$ to $\left.10^{-5} \mathrm{~mol} / \mathrm{L}\right)$. After the cumulative response was stabilized, the rings were washed and allowed to equilibrate to baseline. The procedure was then repeated with an endotheliumindependent vasodilator (acidified $\mathrm{NaNO}_{2}, 10^{-9}$ to $10^{-5} \mathrm{~mol} / \mathrm{L}$ ) to determine smooth muscle function. Endothelial-dependent vasodilator dysfunction was defined as a reduced vasorelaxation in response to $\mathrm{ACh}$ with a normal response to acidified $\mathrm{NaNO}_{2}$.

\section{Quantitation of vascular tissue nitrotyrosine content}

Nitrotyrosine content of thoracic aortic tissue was determined using an enzyme linked immunosorbent assay (ELISA) method described in a recent publication ${ }^{[20]}$. In brief, vascular tissue was separated and homogenized in ice-cold PBS. The homogenates were centrifuged for $10 \mathrm{~min}$ at $12500 \times \mathrm{g}$ at $4{ }^{\circ} \mathrm{C}$. The supernatants were collected and protein concentrations were determined by the bicinchoninic acid (BCA) method. A nitrated protein solution was prepared for use as a standard. These standard samples, along with tissue samples from vas- cular tissue, were applied to disposable sterile ELISA plates and incubated overnight with primary antibody. The secondary antibody was added, and the peroxidase reaction product was generated by using $o$-phenylenediamine dihydrochloride (OPD) solution. The optical density was measured at 460 $\mathrm{nm}$ using a microplate reader (Spectra Max Plus, Molecular Devices Corp, CA, USA). The amount of nitrotyrosine content in tissue samples was calculated using standard curves generated from nitrated BSA containing known amounts of nitrotyrosine and expressed as nmol per mg of protein.

\section{Immunohistological detection of inducible NOS and nitrotyrosine} Thoracic aortic arteries were removed and stored in 10\% formalin for $<48 \mathrm{~h}$. Fixed artery segments were dehydrated and embedded in paraffin, and sections were cut at $5 \mu \mathrm{m}$ and mounted onto glass slides. Immunohistochemical detections of iNOS and nitrotyrosine were performed ${ }^{[20]}$. Rabbit polyclonal antibody against iNOS was purchased from Santa Cruz Biotechnology (Santa Cruz, CA) and rabbit polyclonal antibody against nitrotyrosine was purchased from Upstate Biotechnology (Lake Placid, NY).

\section{Detection of inducible NOS by Western blot}

Thoracic aortic arteries were lysed and homogenated. The protein concentration in the supernatant was detected using BCA kit. Then proteins from tissue homogenate were loaded on sodium dodecyl sulfate-polyacrylamide gel (SDS-PAGE), transferred onto nitrocellulose membrane, blocked with bovine serum albumin, and then incubated using the primary antibody (anti-iNOS or anti- $\beta$-actin). The primary antibodies used were: rabbit polyclonal against iNOS (Santa Cruz, CA), and rabbit polyclonal against $\beta$-actin (Santa Cruz, CA). Specific antibody binding was detected using electrochemiluminescence. The density of the scanned protein bands was measured by image analysis software and the results were presented as \% change of the loading control.

\section{Statistical analysis}

All values in the text and figures are presented as means \pm SD. All data were subjected to one-way ANOVA followed by the Scheffe's correction for post hoc t-test comparison. Probabilities of $P \leq 0.05$ were considered to be statistically significant.

\section{Results}

Aging induced the impairment of endothelial-dependent vasodilation

In aortic rings isolated from the rats, NE-induced pre-contraction levels in the young and aged groups were $(2.5 \pm 0.3) \mathrm{g}$ and $(2.7 \pm 0.4) \mathrm{g}$, respectively. There was no significant difference between the two groups. In aortic rings isolated from young rats, $10^{-9}-10^{-5} \mathrm{~mol} / \mathrm{L} \mathrm{ACh}$ induced a concentration-dependent vasorelaxation. In contrast, aortic rings isolated from aged rats showed a significant right-shifting of their doseresponse curve to $\mathrm{ACh}$ (Figure 1A). Compared to the young group $(95 \% \pm 8 \%)$, the maximal relaxation in the aged group decreased significantly $(70 \% \pm 15 \%, P<0.01)$. The $-\log E_{50}$ val- 

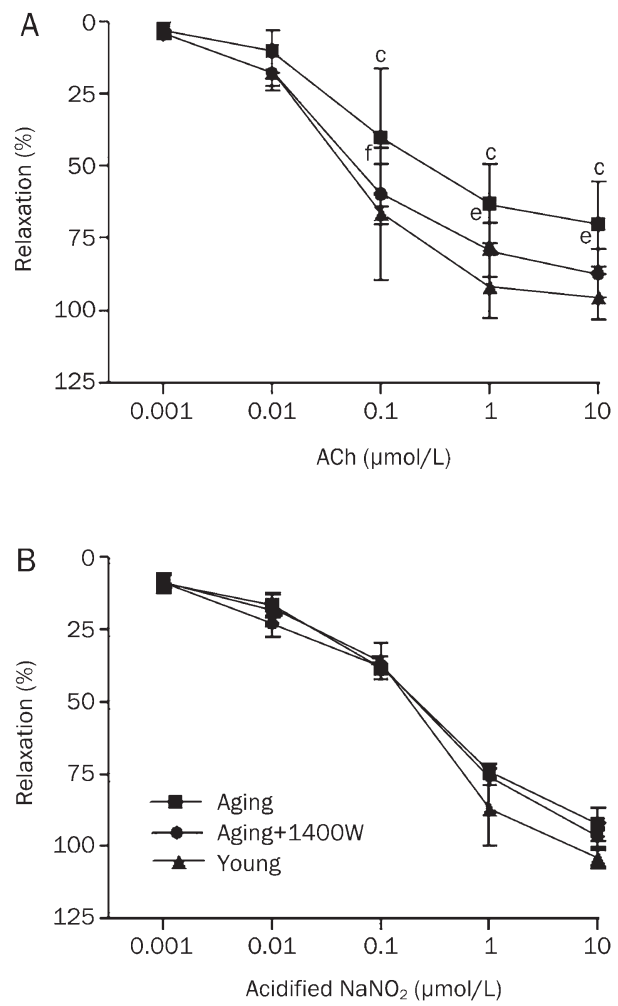

Figure 1. Vasorelaxation responses of isolated thoracic aortic rings to each concentration of acetylcholine (A, ACh, an endothelium-dependent vasodilator) or acidified $\mathrm{NaNO}_{2}$ ( $\mathrm{B}$, an endothelium-independent vasodilator). ${ }^{\mathrm{C}} P<0.01$ vs young group; ${ }^{e} P<0.05,{ }^{\mathrm{f}} P<0.01$ vs aging group. $n=6-8$ rats per group.

ues in the young and aged groups were (7.4 \pm 0.1$)$ and (6.8 \pm 0.5$)$, respectively $(P<0.05)$. While in the young and aged group the maximal relaxation levels induced by $10^{-9}-10^{-5} \mathrm{~mol} / \mathrm{L}$ acidified $\mathrm{NaNO}_{2}$, an exogenous $\mathrm{NO}$ donor, were $(97 \% \pm 4 \%)$ and $(90 \% \pm 6 \%)$, respectively $(P>0.05)$. The $-\log E C_{50}$ values in the young and aged groups were $(6.8 \pm 0.2)$ and $(6.6 \pm 0.5)$, respectively $(P>0.05$, Figure $1 \mathrm{~B})$. These results proved that aging caused endothelium-dependent vasodilator dysfunction.
iNOS was involved in aging-induced impairment of endothelial vasodilator

To determine the role of iNOS in aging-induced endothelium vasodilator dysfunction, firstly we observed iNOS expression by immunohistological and Western blot. As illustrated in Figure 2, compared to the young group, endothelial and smooth muscle expression of iNOS was significantly increased in vascular samples from the aged group. After treatment with the highly selective iNOS inhibitor $1400 \mathrm{~W}$, the expression of iNOS in the endothelial and smooth muscle was significantly decreased compared with the aged group. Then, following treatment with a highly selective iNOS inhibitor $1400 \mathrm{~W}$, endothelial and smooth muscle function in aged rat aortas were determined by comparing the vasorelaxation response to ACh with that of acidified $\mathrm{NaNO}_{2}$. As illustrated in Figure 1, 1400W markedly improved ACh-induced vasorelaxation (maximal relaxation: $87 \% \pm 8 \%, P<0.05,-\log \mathrm{EC}_{50}$ : $7.3 \pm 0.2, P<0.05$ ), while having no effect on acidified $\mathrm{NaNO}_{2}-$ induced vasorelaxation (maximal relaxation: $94 \% \pm 3 \%, P>0.05$, $\left.-\log \mathrm{EC}_{50}: 6.5 \pm 0.3, P>0.05\right)$. These results demonstrated that $1400 \mathrm{~W}$ attenuated aging-induced endothelial dysfunction.

The possible mechanisms of vasculoprotection by $1400 \mathrm{~W}$

To determine the possible mechanisms of $1400 \mathrm{~W}$ vasculoprotection, we investigated the content and expression of nitrotyrosine in aortic vessels of aged rats. As illustrated in Figure $3 \mathrm{~A}$, strong nitrotyrosine staining was detected in endothelial cells and smooth muscle cells from aged rats, and treatment with $1400 \mathrm{~W}$ reduced nitrotyrosine staining. Quantitative ELISA results indicated that a 5.7 -fold increase in nitrotyrosine content was observed in aged tissue, and this increased nitrotyrosine content was markedly reduced by in vivo administration of 1400W (Figure 3B).

\section{Discussion}

The present study confirmed previous reports that aging induces endothelium-dependent vasodilator dysfunction. Moreover, we have also found that iNOS expression increasesd in vascular tissues of aged rats. In addition, we have

\section{A}

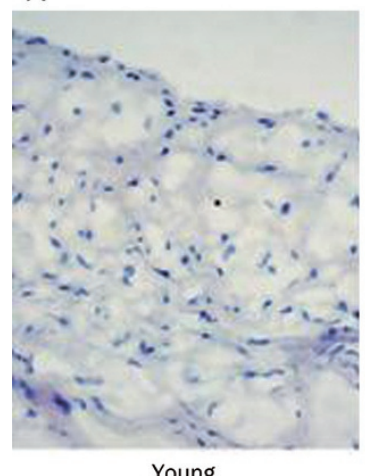

Young

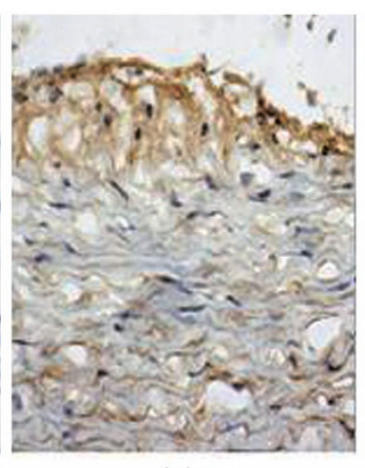

Aging

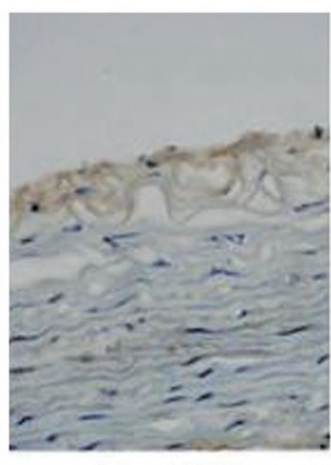

Aging $+1400 \mathrm{~W}$
B
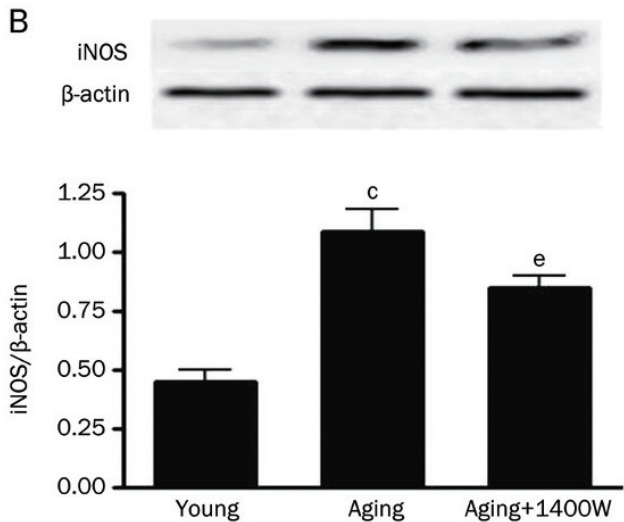

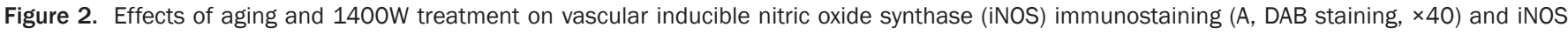
expression (B, Western blot). ${ }^{\mathrm{c}} P<0.01$ vs young group; ${ }^{\mathrm{e}} \mathrm{P}<0.05$ vs aging group. $n=7$ rats per group. 
A

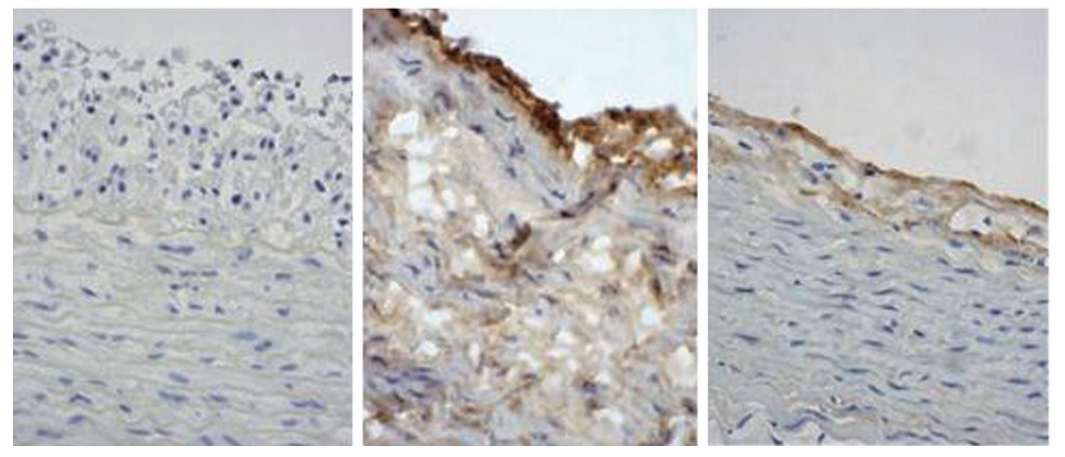

B

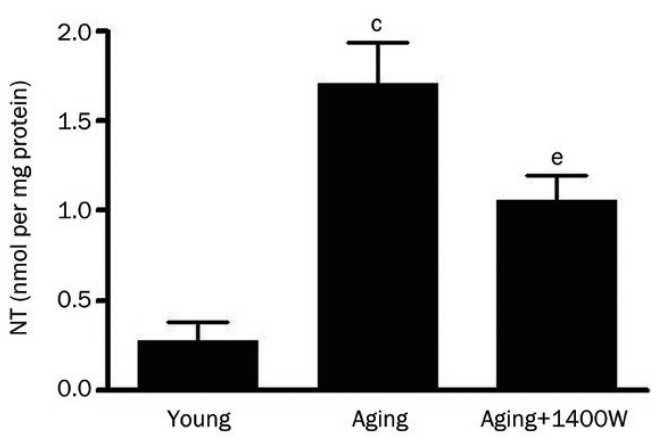

Figure 3. Effect of $1400 \mathrm{~W}$, a selective iNOS inhibitor, on nitrotyrosine (NT) staining (A, DAB staining, $\times 40)$ and vascular nitrotyrosine content $(B)$ in aged rats. ${ }^{c} P<0.01$ vs young group; ${ }^{e} P<0.05$ vs aging group. $n=7$ rats per group.

demonstrated that a selective inhibitor 1400W improves the endothelium-dependent vasodilation possibly by attenuating peroxynitrite formation.

Aging is a major risk factor for cardiovascular disease, and the incidence rate of cardiovascular disease such as hypertension, coronary heart disease and cardiac insufficiency increases with age. A large number of animal experiments and clinical studies have shown that aging induces endothelial vasodilator dysfunction, while the dysfunction of vascular endothelium is closely linked to the occurrence and development of a variety of cardiovascular diseases. Although the specific mechanism of endothelial dysfunction is unclear, protecting endothelial function is the key to prevent subsequent cardiovascular disease. In our present study, ACh-induced maximal relaxation of aortic rings in the aged group was significantly reduced compared to the young group, while acidified $\mathrm{NaNO}_{2}$-induced maximal relaxation had no significant difference between the two groups. These results confirmed that aging induced the decline of endothelium-dependent vasodilation.

A large number of studies suggest that markedly increased $\mathrm{NO}$ and decreased bioavailability is considered to be one of the most important mechanisms that results in endothelial dysfunction $^{[22]}$. Endogenous $\mathrm{NO}$ is generated from $L$-arginine by three NOS, including neuronal-type (nNOS) from the central nervous system, iNOS from mammalian nucleated cells and eNOS from endothelial cells. There are two types of NOS in vascular tissue, eNOS and iNOS. In the physiological state $\mathrm{NO}$ is at a low concentration (about $0.1-100 \mathrm{nmol} / \mathrm{L}$ ) and mainly generated by eNOS, but in the pathological state it is mainly produced by iNOS and maintains a relatively high level ${ }^{[23-25]}$.

Decreased eNOS and endogenous NO was considered to be a key factor of endothelial dysfunction. However, in recent few years, several studies have shown that iNOS expression is significantly increased in vascular tissue of the aged rats, which suggested that increased iNOS may also be involved in endothelial dysfunction induced by aging. But the existing research ${ }^{[15,26]}$ with non-specific NOS inhibitor treatment could not clarify whether iNOS was directly related to endothelial dysfunction induced by aging. Therefore, we used the highly selective iNOS inhibitor $1400 \mathrm{~W}$ in our present study. It has been reported that $1400 \mathrm{~W}$ inhibited iNOS activity by binding with iNOS ${ }^{[27]}$, but some studies found that $1400 \mathrm{~W}$ could decrease iNOS expression ${ }^{[28,29]}$. In this study, we also found that iNOS expression was increased significantly in vascular tissue of aged rats. When treated with $1400 \mathrm{~W}$, iNOS expression was decreased and endothelial vasodilator dysfunction was significantly improved. These results demonstrated that iNOS was directly involved in endothelial dysfunction induced by aging.

It has been shown that an imbalance in the ratio of nitric oxide to superoxide anion due to a prevalence of the superoxide anion leads to an alteration in vascular reactivity ${ }^{[30]}$. Several studies have found that iNOS triggered a significant increased NO production, which could create a nitrative stress and generate the toxic oxidant molecule peroxynitrite $\left(\mathrm{ONOO}^{-}\right)$excessively ${ }^{[31]}$. $\mathrm{ONOO}^{-}$is responsible for nitration of tyrosine residues in proteins, therefore the presence of nitrotyrosine (NT) in plasma proteins is considered an indirect evidence of $\mathrm{ONOO}^{-}$production $^{[30]}$. Our studies confirmed increased expression of NT in the aged rat vessels, and such increase was blocked markedly by $1400 \mathrm{~W}$, a highly selective iNOS inhibitor. This result revealed that decreased $\mathrm{ONOO}^{-}$ could be the reason that $1400 \mathrm{~W}$ is vasculoprotective, but to confirm it we should perform further studies.

In summary, the findings demonstrated with a highly selective iNOS inhibitor $1400 \mathrm{~W}$ improved the endotheliumdependent vasorelaxation in aged animals. Though our study has some limitations, it strongly suggests that we can improve endothelial vasodilation in aged rats by inhibiting iNOS, and further reduce the incidence of age-related cardiovascular diseases.

\section{Acknowledgements}

This research is supported partially by National Natural Science Foundation of China (30973163) to Hui-rong LIU.

\section{Author contribution}

Hui-rong LIU designed the research; Jue TIAN and Lin QUAN detected endothelial function and performed Western blot, 
Ke WANG and Xiu-rui MA detected nitrotyrosine content, Li GUO and Jing-yi LIU performed immunohistological staining; Jin WANG, Ye WU analyzed data; Jue TIAN, Zi YAN wrote the paper; Su-li ZHANG, Lin ZUO corrected this paper.

\section{References}

1 Lakatta EG, Levy D. Arterial and cardiac aging: major shareholders in cardiovascular disease enterprises: Part I: aging arteries: a "set up" for vascular disease. Circulation 2003; 107: 139-46.

2 Lakatta EG, Levy D. Arterial and cardiac aging: major shareholders in cardiovascular disease enterprises: Part II: the aging heart in health: links to heart disease. Circulation 2003; 107: 346-54.

3 Lakatta EG. Arterial and cardiac aging: major shareholders in cardiovascular disease enterprises: Part III: cellular and molecular clues to heart and arterial aging. Circulation 2003; 107: 490-7.

4 Sayer AA, Cooper C. The ageing process. Menopause Int 2000; 6: 55-65.

5 Behnke BJ, Delp MD. Aging blunts the dynamics of vasodilation in isolated skeletal muscle resistance vessels. J Appl Physiol 2010; 108: 14-20.

6 Nair N, Oka RK, Waring LD, Umoh EM, Taylor CB, Cooke JP. Vascular compliance versus flow-mediated vasodilation: correlation with cardiovascular risk factors. Vasc Med 2005; 10: 275-83.

7 Cannon RO 3rd. Role of nitric oxide in cardiovascular disease: focus on the endothelium. Clin Chem 1998; 44: 1809-19.

8 Panza JA, Casino PR, Kilcoyne CM, Quyyumi AA. Role of endotheliumderived nitric oxide in the abnormal endothelium-dependent vascular relaxation of patients with essential hypertension. Circulation 1993; 87: 1468-74.

9 Casino PR, Kilcoyne CM, Quyyumi AA, Hoeg JM, Panza JA. The role of nitric oxide in endothelium-dependent vasodilation of hypercholesterolemic patients. Circulation 1993; 88: 2541-7.

10 Förstermann U, Boissel JP, Kleinert H. Expressional control of the 'constitutive' isoforms of nitric oxide synthase (NOS I and NOS III). FASEB J 1998; 12: 773-90.

11 Shah AM. Inducible nitric oxide synthase and cardiovascular disease. Cardiovasc Res 2000; 45: 148-55.

12 Liu VW, Huang PL. Cardiovascular roles of nitric oxide: a review of insights from nitric oxide synthase gene disrupted mice. Cardiovasc Res 2008; 77: 19-29.

13 Barton M, Cosentino F, Brandes RP, Moreau P, Shaw S, Lüscher TF. Anatomic heterogeneity of vascular aging: role of nitric oxide and endothelin. Hypertension 1997; 30: 817-24.

14 Tanabe T, Maeda S, Miyauchi T, lemitsu M, Takanashi M, IrukayamaTomobe $\mathrm{Y}$, et al. Exercise training improves ageing-induced decrease in eNOS expression of the aorta. Acta Physiol Scand 2003; 178: 3-10.

15 Csiszar A, Ungvari Z, Edwards JG, Kaminski P, Wolin MS, Koller A, et al. Aging-induced phenotypic changes and oxidative stress impair coronary arteriolar function. Circ Res 2002; 90: 1159-66.

16 Cernadas MR, Sánchez de Miguel L, García-Durán M, GonzálezFernández $\mathrm{F}$, Millás I, Montón $\mathrm{M}$, et al. Expression of constitutive and inducible nitric oxide synthases in the vascular wall of young and aging rats. Circ Res 1998; 83: 279-86.

17 Song MY, Zwemer CF, Whitesall SE, D'Alecy LG. Acute and conditioned hypoxic tolerance augmented by endothelial nitric oxide synthase inhibition in mice. J Appl Physiol 2007; 102: 610-5.

18 Lasley RD, Keith BJ, Kristo G, Yoshimura Y, Mentzer RM Jr. Delayed adenosine A1 receptor preconditioning in rat myocardium is MAPK dependent but iNOS independent. Am J Physiol Heart Circ Physiol 2005; 289: H785- H791.

19 Salloum F, Yin C, Xi L, Kukreja RC. Sildenafil induces delayed preconditioning through inducible nitric oxide synthase-dependent pathway in mouse heart. Circ Res 2003; 92: 595-7.

20 Ma XL, Gao F, Nelson AH, Lopez BL, Christopher TA, Yue TL, et al. Oxidative inactivation of nitric oxide and endothelial dysfunction in stroke-prone spontaneous hypertensive rats. J Pharmacol Exp Ther 2001; 298: 879-85.

21 Liu YH, You Y, Song T, Wu SJ, Liu LY. Impairment of endotheliumdependent relaxation of rat aortas by homocysteine thiolactone and attenuation by captopril. J Cardiovasc Pharmacol 2007; 50: 155-61.

22 Herrera MD, Mingorance C, Rodríguez-Rodríguez R, Alvarez de Sotomayor M. Endothelial dysfunction and aging: an update. Ageing Res Rev 2010; 9: 142-52.

23 Beckman JS, Koppenol WH. Nitric oxide, superoxide, and peroxynitrite: the good, the bad, and ugly. Am J Physiol 1996; 271: C1424-C1437.

24 Boyd CS, Cadenas E. Nitric oxide and cell signaling pathways in mitochondrial-dependent apoptosis. Biol Chem 2002; 383: 411-23.

25 Fierro IM, Nascimento-DaSilva V, Arruda MA, Freitas MS, Plotkowski $\mathrm{MC}$, Cunha FQ, et al. Induction of NOS in rat blood PMN in vivo and in vitro: modulation by tyrosine kinase and involvement in bactericidal activity. J Leukoc Biol 1999; 65: 508-14.

26 Li QX, Xiong ZY, Hu BP, Tian ZJ, Zhang HF, Gou WY, et al. Agingassociated insulin resistance predisposes to hypertension and its reversal by exercise: the role of vascular vasorelaxation to insulin. Basic Res Cardiol 2009; 104: 269-84.

27 Garvey EP, Oplinger JA, Furfine ES, Kiff RJ, Laszlo F, Whittle BJ, et al. 1400W is a slow, tight binding, and highly selective inhibitor of inducible nitric-oxide synthase in vitro and in vivo. J Biol Chem 1997; 272: 4959-63.

28 El-Mas MM, Fan M, Abdel-Rahman AA. Endotoxemia-mediated induction of cardiac inducible nitric-oxide synthase expression accounts for the hypotensive effect of ethanol in female rats. J Pharmacol Exp Ther 2008; 324: 368-75.

29 Kan WH, Hsu JT, Schwacha MG, Choudhry MA, Raju R, Bland KI, et al. Selective inhibition of iNOS attenuates trauma-hemorrhage/ resuscitation-induced hepatic injury. J Appl Physiol 2008; 105: 107682.

30 Ceriello A. Nitrotyrosine: new findings as a marker of postprandial oxidative stress. Int J Clin Pract Suppl 2002; 129: 51-8.

31 Li D, Qu Y, Tao L, Liu H, Hu A, Gao F, et al. Inhibition of iNOS protects the aging heart against beta-adrenergic receptor stimulation-induced cardiac dysfunction and myocardial ischemic injury. J Surg Res 2006; 131: $64-72$. 